\title{
TARGETLESS CAMERA CALIBRATION
}

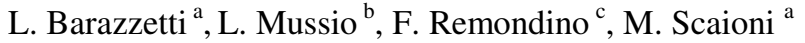 \\ ${ }^{a}$ Politecnico di Milano, Dept. of Building Engineering Science and Technology, Milan, Italy \\ \{luigi.barazzetti, marco.scaioni\}@ polimi.it, web: http://www.icet-rilevamento.lecco.polimi.it/ \\ ${ }^{\mathrm{b}}$ Politecnico di Milano, Dept. of Environmental, Hydraulic, Infrastructures and Surveying Engineering, Milan, Italy \\ luigi.mussio@polimi.it, web: http://www.diiar.polimi.it/diiar \\ c 3D Optical Metrology Unit, Bruno Kessler Foundation (FBK), Trento, Italy \\ remondino@fbk.eu, web: http://3dom.fbk.eu
}

\section{KEY WORDS: Automation, Accuracy, Calibration, Matching, Orientation}

\begin{abstract}
:
In photogrammetry a camera is considered calibrated if its interior orientation parameters are known. These encompass the principal distance, the principal point position and some Additional Parameters used to model possible systematic errors. The current state of the art for automated camera calibration relies on the use of coded targets to accurately determine the image correspondences. This paper presents a new methodology for the efficient and rigorous photogrammetric calibration of digital cameras which does not require any longer the use of targets. A set of images depicting a scene with a good texture are sufficient for the extraction of natural corresponding image points. These are automatically matched with feature-based approaches and robust estimation techniques. The successive photogrammetric bundle adjustment retrieves the unknown camera parameters and their theoretical accuracies. Examples, considerations and comparisons with real data and different case studies are illustrated to show the potentialities of the proposed methodology.
\end{abstract}

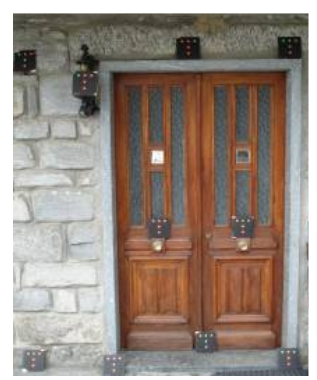

a)
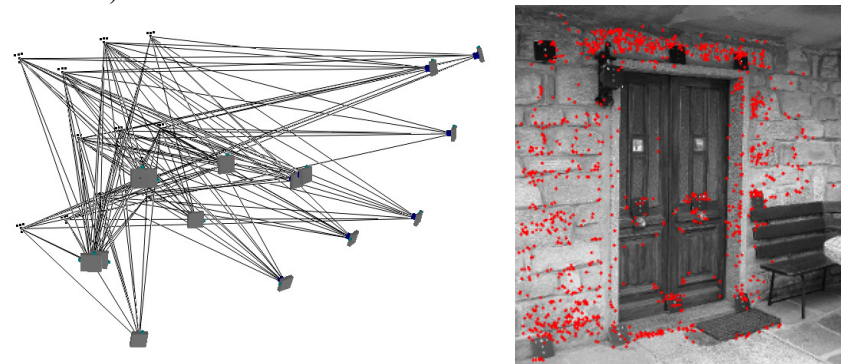

b)

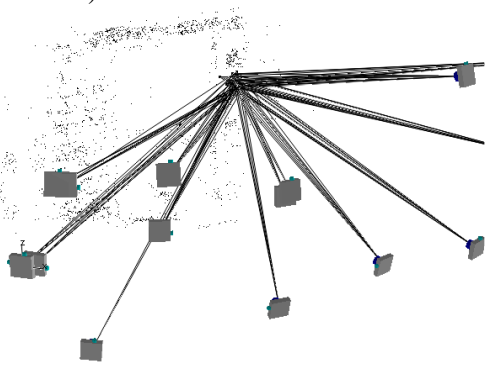

Figure 1. A target-based calibration procedure (a) and the targetless approach (b).

\section{INTRODUCTION}

Accurate camera calibration and image orientation procedures are a necessary prerequisite for the extraction of precise and reliable 3D metric information from images (Gruen and Huang, 2001). A camera is considered calibrated if its principal distance, principal point offset and lens distortion parameters are known. Camera calibration has always been an essential component of photogrammetric measurement. Self-calibration is nowadays an integral and routinely applied operation within photogrammetric image triangulation, especially in highaccuracy close-range measurement. With the very rapid growth in adoption of off-the-shelf (or consumer-grade) digital cameras for 3D measurement applications, however, there are many situations where the geometry of the image network cannot support the robust recovery of camera interior parameters via on-the-job calibration. For this reason, stand-alone and targetbased camera calibration has again emerged as an important issue in close-range photogrammetry.

In many applications, especially in Computer Vision (CV), only the focal length is generally recovered. In case of precise photogrammetric measurements, the whole set of calibration parameters is instead employed. Various algorithms for camera calibration have been reported over the past years in the photogrammetry and CV literature (Remondino and Fraser, 2006). The algorithms are usually based on perspective or projective camera models, with the most popular approach being the well-known self-calibrating bundle adjustment (Brown, 1976; Fraser, 1997; Gruen and Beyer, 2001). It was first introduced in close-range photogrammetry in the early 1970s by Brown (1971). Analytical camera calibration was a major topic of research interest in photogrammetry over the next decade and it reached its full maturity in the mid 1980s. In the early days of digital cameras, self-calibration became again a hot research topic and it reached its maturity in the late ' 90 s with the development of fully automated vision metrology systems mainly based on targets (e.g. Ganci and Handley, 1998). In the last decade, with the tremendous use of consumergrade digital cameras for many measurement applications, there was a renewed interest in stand-alone photogrammetric calibration approaches, especially for fully automatic on-the-job calibration procedures. Nowadays the state of the art basically relies on the use of coded targets which are depicted in images forming a block with a suitable geometry for estimating all the calibration parameters (Cronk et al., 2006). Target measurement and identification is performed in an automatic way. A bundle 
adjustment allows then the estimation of all the unknown parameters and their theoretical accuracies.

On the other hand, camera calibration continues to be a more active area of research within the $\mathrm{CV}$ community, with a perhaps unfortunate characteristic of much of the work being that it pays too little heed to previous findings from photogrammetry. Part of this might well be explained in terms of a lack of emphasis on (and interest in) accuracy aspects and a basic premise that nothing whatever needs to be known about the camera which is to be calibrated within a linear projective rather than Euclidean scene reconstruction.

\section{CAMERA CALIBRATION IN PHOTOGRAMMETRY AND COMPUTER VISION}

In photogrammetry camera calibration is meant as the recovery of the interior camera parameters. Camera calibration plays a fundamental role in both photogrammetry and CV but there is an important distinction between the approaches used in both disciplines. Even the well-known term self-calibration has different meanings.

Lens distortion generates a misalignment between the perspective centre, the image point and the object point. It is quite simple to understand that the collinearity principle, which is the basis for image orientation, is no longer respected ("departure from collinearity"). Modelling lens distortion allows to strongly reduce this effect. A calibrated camera is a powerful measuring tool, with a precision superior to $1: 25,000$ as reported in different vision metrology applications (Maas and Niederöst, 1997; Albert et al., 2002; Amiri Parian et al., 2006; Barazzetti and Scaioni, 2009; Barazzetti and Scaioni, 2010). The importance of camera calibration is confirmed by the vast number of papers in the technical literature: accuracy aspects, low-cost and professional cameras, stability and behaviour of the parameters, variations in the different colour channels as well as algorithmic issues were reported in Fraser and Shortis (1995), D’Apuzzo and Maas (1999), Läbe and Förstner (2004), Fraser and Al-Ajlouni (2006), Peipe and Tecklenburg (2006) and Remondino and Fraser (2006).

During a photogrammetric camera calibration procedure, the systematic errors in digital CCD/CMOS sensor are universally compensated with an 8-terms physical mathematical model originally formulated by Brown (1971). This comprises terms for the principal distance $(c)$ and principal point offset $\left(x_{0}, y_{0}\right)$ correction, three coefficients for the radial distortion $\left(k_{1}, k_{2}, k_{3}\right)$, and two coefficients for the decentring distortion $\left(p_{1}, p_{2}\right)$. The model can be extended by two further parameters to account for affinity and shear within the image plane, but such terms are rarely if ever significant in modern digital cameras, especially for heritage and architectural applications. The corrections terms are generally called Additional Parameters (APs).

The three APs used to model the radial distortion $\delta r$ are generally expressed with an odd-ordered polynomial series:

$$
\delta r=k_{1} r^{3}+k_{2} r^{5}+k_{3} r^{7}
$$

where $r$ is the radial distance of the generic image point $(x, y)$ from the principal point $\left(x_{0}, y_{0}\right)$ :

$$
r=\sqrt{\left(x-x_{0}\right)^{2}+\left(y-y_{0}\right)^{2}}
$$

The components along $x$ and $y$ of $\delta r$ may be estimated as follows:

$$
\delta x=\left(x-x_{0}\right) \frac{\delta r}{r}, \quad \delta y=\left(y-y_{0}\right) \frac{\delta r}{r}
$$

The coefficients $k_{i}$ are a function of the used principal distance and are usually highly correlated, with most of the error signal generally being accounted for by the cubic term $k_{1} r^{3}$. The $k_{2}$ and $k_{3}$ terms are typically included for photogrammetric (low distortion) and wide-angle lenses and in higher-accuracy vision metrology applications. Recent research has demonstrated the feasibility of empirically modelling radial distortion throughout the magnification range of a zoom lens as a function of the focal length written to the image EXIF header (Fraser and AlAjlouni, 2006).

A misalignment of the lens elements along the optical axis instead generates decentring distortion. The corrections terms for the measured image coordinates are given by:

$$
\begin{aligned}
& \delta t_{x}=p_{1}\left[r^{2}+2\left(x-x_{0}\right)^{2}\right]+2 p_{2}\left(x-x_{0}\right)\left(y-y_{0}\right) \\
& \delta t_{y}=p_{2}\left[r^{2}+2\left(y-y_{0}\right)^{2}\right]+2 p_{1}\left(x-x_{0}\right)\left(y-y_{0}\right)
\end{aligned}
$$

The decentering distortion parameters $p_{1}$ and $p_{2}$ are invariably strongly projectively coupled with $x_{0}$ and $y_{0}$. Decentering distortion is usually an order of magnitude or more less than the radial distortion and it also varies with focus, but to a much less extent.

Considering all the APs, the image coordinates correction terms can be formulated as:

$$
\begin{aligned}
& \Delta x=-\Delta x_{0}+\frac{x-x_{0}}{c} \Delta c+\delta x+\delta t_{x} \\
& \Delta y=-\Delta y_{0}+\frac{y-y_{0}}{c} \Delta c+\delta x+\delta t_{y}
\end{aligned}
$$

The simultaneous estimation of APs and camera parameters is generally referred to as self-calibrating bundle adjustment. The bundle adjustment with APs needs a favourable network geometry to be correctly solved i.e. convergent and rotated images of a preferably 3D object should be acquired, with well distributed points throughout the image format (Figure 2a). If the network is geometrically weak, high correlations between the unknown parameters may lead to instabilities in the leastsquares estimation. The inappropriate use of the APs can also weaken the bundle adjustment solution, leading to overparameterization, in particular in the case of minimally constrained adjustments (Fraser, 1982).

The collinearity model and the related bundle adjustment problem must be linearized to obtain a system of linear observation equations. The linearized model can be solved with the Gauss-Markov model of least squares (Mikhail et al., 2001) and its solution is rigorous in a functional and stochastic sense. Good initial values of the unknown parameters are needed for the linearization process based on the Taylor series expansion. External constraints (e.g. GNSS/INS data, GCPs) can also be efficiently incorporated into the general model. The final system is made up of observation equations (those written as functions of both observations and parameters) and constraint equations (those written in terms of the parameters). The second group of equations is usually formulated as pseudo-observation equations, where the unknown parameters are linked to their measured values. All the variables in the adjustment become weighted observations. By properly tuning each weight it is possible to give more or less emphasis to the observed values of each unknown parameter. 
If the observations are image coordinates, the reconstruction is affected by an overall ambiguity (i.e. a 3D similarity transformation). This "datum problem" (or rank deficiency) can be solved by introducing ground control points (GCPs) and/or GNSS/INS information. The second solution is almost the standard in aerial photogrammetry, while these data are not generally available in close-range surveys. The rank deficiency of the Least Squares problem can also be removed with an inner constraint. This does not involve external observations and leads to the so-called free-net solution (Granshaw, 1980; Dermanis, 1994). The theoretical accuracy obtainable with a free-net adjustment, coupled with precise image points and good calibration parameters is superior to $1: 100,000$. In some cases, a theoretical accuracy of about one part in a million was reached (Fraser, 1992).

(a)

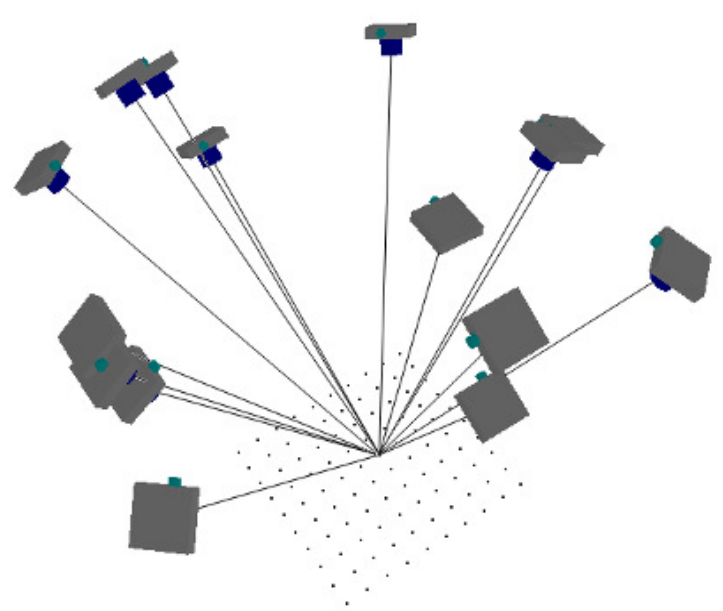

(b)

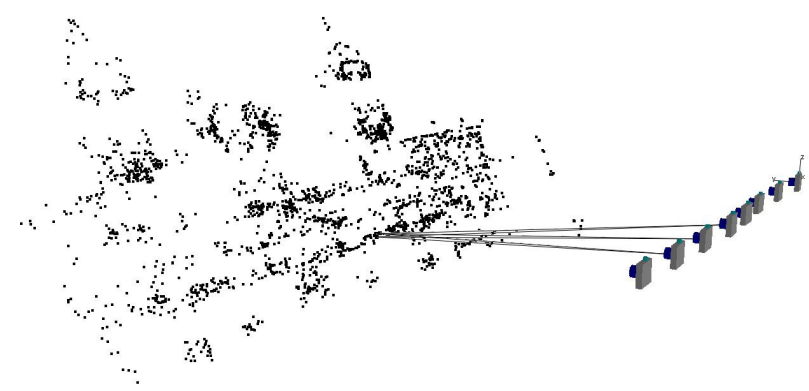

Figure 2. An appropriate image network which allows the correct estimation of all calibration parameters (a). An image network inappropriate for camera calibration and more efficient for scene reconstruction and 3D modeling applications (b).

Some good and practical rules for camera calibration can be summarized as follows:

- self-calibration is only reliable when the image network geometry is favourable, i.e. the camera station configuration comprises highly convergent images, orthogonal roll angles and a large number of well distributed object points. A compensation for departures from collinearity might well be achieved in a bundle adjustment with APs for a weak network, but the precise and reliable recovery of representative calibration values is less likely to be obtained;

- a planar object point array could be employed for camera calibration if the images are acquired with orthogonal roll angles, a high degree of convergence and, desirably, varying object distances. What is sought is the maximum possible imaging scale variation throughout the image format.

- orthogonal roll angles must be present to break the projective coupling between IO and EO parameters. Although it might be possible to achieve this decoupling without $90^{\circ}$ image rotations, through provision of a strongly $3 \mathrm{D}$ object point array, it is always recommended to have 'rolled' images in the self-calibration network;

- the accuracy of a network increases with increasing convergence angles for the imagery. Increasing the angles of convergence also implicitly means increasing the base-todepth $(\mathrm{B} / \mathrm{D})$ ratio.

In $\mathrm{CV}$, the orientation procedures are (often) uncalibrated. This allows one to deal with simple, quick, and flexible acquisition procedures. The mapping between object $(\mathbf{X})$ and image $(\mathbf{x})$ points with the general projective camera model may be written as (Hartley and Zissermann, 2004):

$$
\mathbf{X}=\mathbf{P X}=\left[\begin{array}{ccc}
f & 0 & p_{x} \\
0 & f & p_{y} \\
0 & 0 & 1
\end{array}\right][\mathbf{R}, \mathbf{t}] \mathbf{X}=\mathbf{K}[\mathbf{R}, \mathbf{t}] \mathbf{X}
$$

The matrix $\mathbf{K}$ is the calibration matrix, here written for CCD or CMOS sensors with square pixels and a skew parameter equal to 0 . This relationship includes the focal length $f$ and the principal point offset, so that the model is equivalent to that with interior orientation parameters (although the focal length is not the principal distance, except for lenses focused at infinity). To compensate for the image distortion, a distortion factor $L(r)$ is generally considered, that depends on the radius only (Hartley and Zissermann, 2004):

$$
\begin{aligned}
& x_{\text {corr }}=x_{c}+L(r)\left(x-x_{c}\right) \\
& y_{\text {corr }}=y_{c}+L(r)\left(y-y_{c}\right)
\end{aligned}
$$

where $\left(x_{\text {corr }}, y_{\text {corr }}\right)$ are the corrected (or undistorted) coordinates, and $\left(x_{\mathrm{c}}, y_{\mathrm{c}}\right)$ is the centre of radial distortion.

For the solution of Eq. 6 (based on the corrected image coordinates), a bundle adjustment generally based on the Levenberg-Marquard algorithm is employed. Given a set of $n$ image points and $m$ 3D points, the reprojection error (i.e., the distance between the back projected $3 \mathrm{D}$ point and the corresponding measured image point) is minimized (Triggs et al., 2000):

$\min \sum_{i=1}^{n} \sum_{j=1}^{m} w_{i j}\left\|\mathbf{x}_{i j}-\mathbf{P}_{i}\left(\mathrm{~K}_{i}, \mathbf{X}_{j}\right)\right\|^{2}$

where $\mathbf{x}_{i j}$ is the measured image point, $\mathbf{K}_{i}$ are the camera parameters, $\mathbf{P}_{i}$ is the projection matrix, $\mathbf{X}_{j}$ is a $3 \mathrm{D}$ point. The coefficient $w_{i j}$ is set to 1 if camera $i$ observes point $j$, and 0 otherwise.

In CV applications all camera parameters (interior and exterior) are usually recovered simultaneously and directly from the same set of images employed to reconstruct the scene. This solution is acceptable if the main goal is not an accurate metric reconstruction. On the other hand, in photogrammetric applications, although the estimation of the camera parameters is still carried out within a bundle adjustment, the network geometry used for object reconstruction is generally not sufficient to estimate the interior parameters at the same time (Figure 2). Therefore, it is strongly suggested to separate the 
recovery of the interior and exterior orientation parameters by means of two separate procedures with adequate networks.

\section{TARGETLESS CAMERA CALIBRATION}

Since many years commercial photogrammetric packages use coded targets for the automated calibration and orientation phase (Ganci and Handley, 1998; Cronk et al., 2006). Coded targets can be automatically recognized, measured and labelled to solve for the identification of the image correspondences and the successive camera parameters within few minutes.
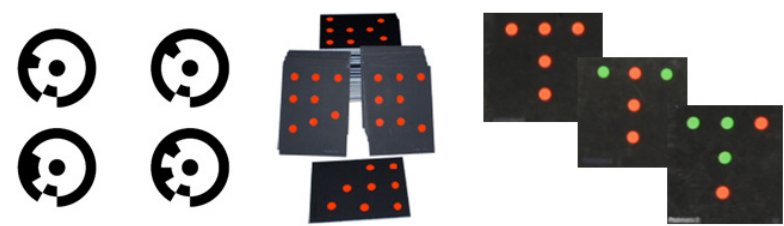

Figure 3. Examples of coded targets.

Commercial software (e.g., iWitness and PhotoModeler) typically works with small coded targets (Figure 3) that can be distributed in order to form a $3 \mathrm{D}$ calibration polygon. The main advantage of this procedure is related to the possibility to have a portable solution. This is useful in many photogrammetric surveys and to assure a correct and automated identification of the image correspondences.

This paper presents a new methodology to efficiently calibrate a digital camera using the ATiPE system, widely described in Barazzetti et al. (2010a) and Barazzetti (2011). ATiPE can automatically and accurately identify homologues points from a set of convergent images without any coded target or marker. A set of natural features of an existing object are used to determine the image correspondences. These image points are automatically matched with the implemented feature-based matching (FBM) approaches. The object should have a good texture in order to provide a sufficient number of tie points well distributed in the images. The operator has to acquire a set of images (12-15) with a good spatial distribution around an object (including $90^{\circ}$ camera roll variations). Architectural objects (e.g. arcades, building facades, colonnades and similar) should be avoided because of their repetitive textures and symmetries. Big rocks, bas-reliefs, decorations, ornaments or even a pile of rubble are appropriate (Figure 4).
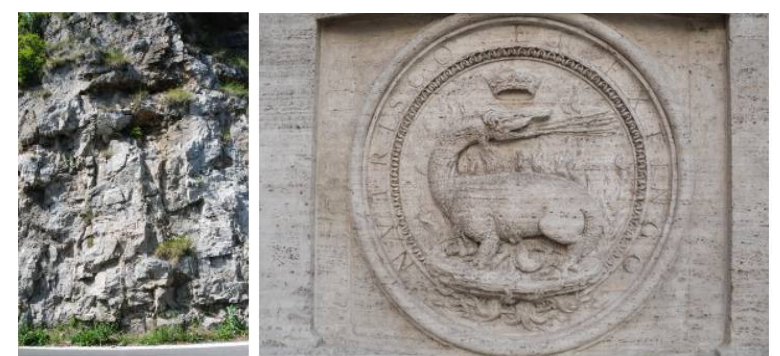

Figure 4. Examples of good calibration objects.

ATiPE uses SIFT (Lowe, 2004) and SURF (Bay et al., 2008) as feature detectors and descriptors. A kd-tree search (Arya et al., 1998) speeds up the comparison between the descriptors of the adopted FBM algorithms. The experimental tests demonstrated that points are rarely matched with convergence angles superior to $30-40^{\circ}$. A normal exhaustive quadratic comparison of the feature descriptors is a more robust approach in case of very convergent images. This is the most important drawback of the method, which leads to a long elaboration time. The global processing time is often unpredictable. It ranges from few minutes up to some hours for large datasets with very convergent images.

\section{EXAMPLES AND PERFORMANCE ANALYSES}

\subsection{Practical tests}

Figure 5a shows a calibration testfield created with some coded targets. 16 images are acquired using a Nikon D700 $(4,256 \times 2,832$ pixels $)$ equipped with a $35 \mathrm{~mm}$ Nikkor lens (focused at $\infty$ ). The camera calibration solution was computed within Australis, which can automatically detected all the coded targets and compute the calibration parameters (Table 1) according to the 8-term mathematical model described in Section 2. The same images were then processed with ATiPE in order to extract a set of natural points randomly distributed in the scene (Figure 5b). The successive estimation of the bundle solution (within Australis) provided all calibration parameters with the camera poses and 3D points (Figure 5c and 5d). The corresponding calibration parameters and their precisions are equal and shown in Table 1. The project with targets comprehends $553 \mathrm{D}$ points $(5$ circles $\times 11$ targets $)$. The estimated theoretical accuracies along the $x, y$ and $z$ axis were $1: 83,000,1: 40,900$ and $1: 64,500$, respectively. The processing with ATiPE provided for 2,531 3D natural points (at least 4 images for each point; 1,356 points were matched in 6 or more images). The estimated theoretical accuracies along the $x, y$ and $z$ axis were $1: 22,100,1: 6,500 ; 1: 9,400$, respectively. This disparity is motivated by the use of different matching strategies leading to different accuracy in the image measurements. The measurement of the centre of the targets is performed more precisely than natural points extracted using FBM methods. Indeed, as also stated by Fraser (1996), the accuracy of the computed object coordinates depends on the image measurement precision, image scale and geometry as well as the number of exposure.

\begin{tabular}{|c|c|c|}
\hline & \multicolumn{2}{|c|}{ Targets } \\
\hline & Value & Std.dev \\
\hline$R M S E(\mu m)$ & 0.89 & \\
\hline$c(\mathrm{~mm})$ & 35.8970 & \pm 0.005 \\
\hline$x_{0}(\mathrm{~mm})$ & -0.0973 & \pm 0.004 \\
\hline$y_{0}(\mathrm{~mm})$ & -0.1701 & \pm 0.004 \\
\hline$k_{1}$ & $6.97946 \mathrm{e}-005$ & $\pm 6.0510 \mathrm{e}-007$ \\
\hline$k_{2}$ & $-5.40867 e-008$ & $\pm 3.2964 \mathrm{e}-009$ \\
\hline$k_{3}$ & $-1.21579 \mathrm{e}-011$ & $\pm 5.4357 \mathrm{e}-012$ \\
\hline$p_{1}$ & $-1.4449 \mathrm{e}-006$ & $\pm 1.074 \mathrm{e}-006$ \\
\hline \multirow[t]{3}{*}{$p_{2}$} & $-5.3279 \mathrm{e}-006$ & $\pm 9.990 \mathrm{e}-007$ \\
\hline & \multicolumn{2}{|c|}{$A T i P E$} \\
\hline & Value & Std.dev \\
\hline$R M S E(\mu m)$ & 2.97 & \\
\hline$c(m m)$ & 35.8891 & \pm 0.003 \\
\hline$x_{0}(\mathrm{~mm})$ & -0.0703 & \pm 0.002 \\
\hline$y_{0}(\mathrm{~mm})$ & -0.1716 & \pm 0.002 \\
\hline$k_{1}$ & $6.97850 \mathrm{e}^{-5}$ & $\pm 3.6472 \mathrm{e}^{-7}$ \\
\hline$k_{2}$ & $-5.64321 e^{-8}$ & $\pm 2.0338 \mathrm{e}^{-9}$ \\
\hline$k_{3}$ & $-5.65025 \mathrm{e}^{-12}$ & $\pm 3.4521 \mathrm{e}^{-12}$ \\
\hline$p_{1}$ & $-6.3586 \mathrm{e}^{-6}$ & $\pm 6.064 \mathrm{e}^{-7}$ \\
\hline$p_{2}$ & $-5.2057 \mathrm{e}^{-6}$ & $\pm 6.068 \mathrm{e}^{-7}$ \\
\hline
\end{tabular}

Table 1. Camera interior parameters estimated with the target-based approach and using ATiPE (targetless) method for a Nikon D700 mounting a $35 \mathrm{~mm}$ lens. 

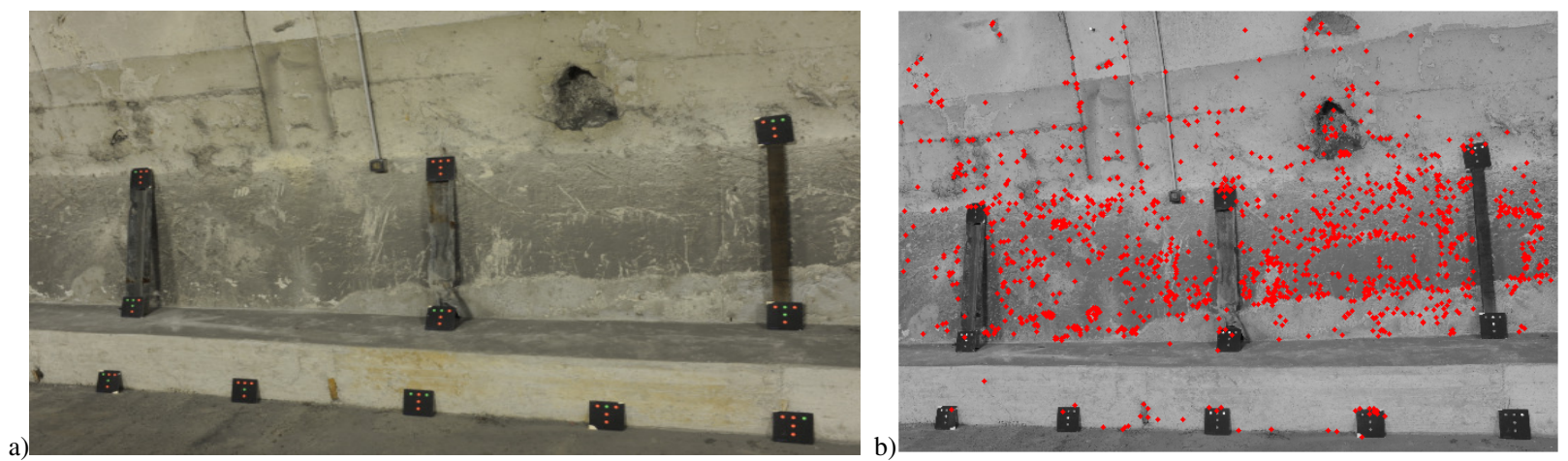

$x$

c)
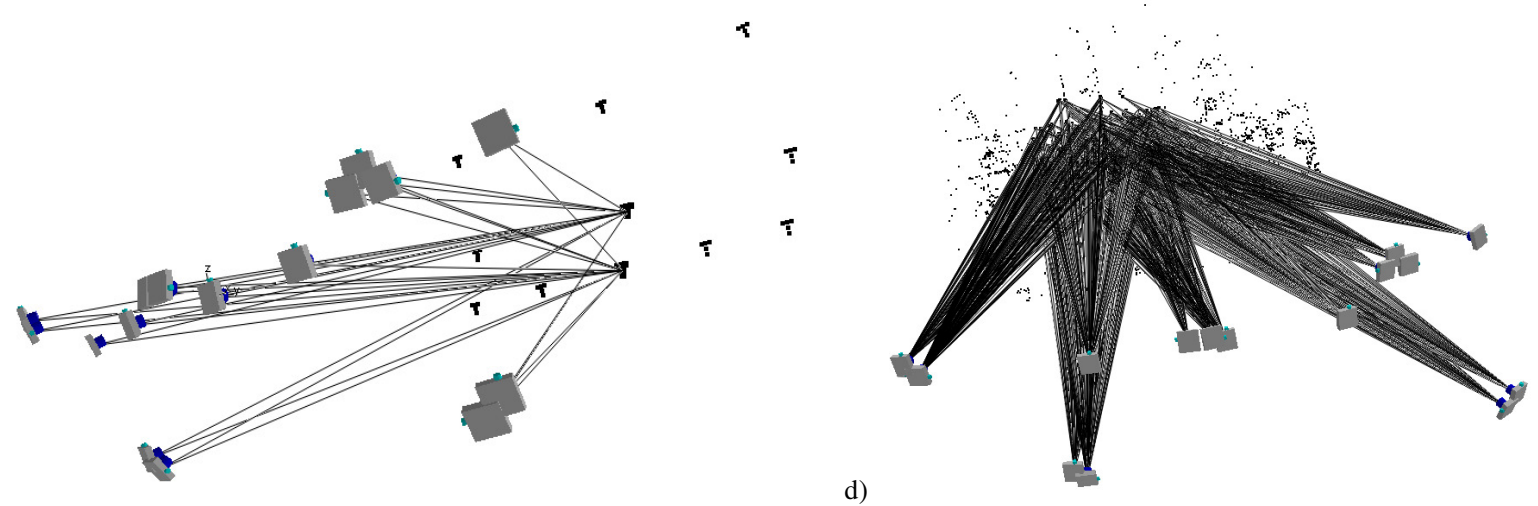

Figure 5. The calibration polygon with iWitness/Australis targets (a). Tie points extracted by ATiPE using the natural texture of the scene (b). The bundle adjustment results achieved in Australis using coded target image coordinates (c) and natural features image coordinates (d). The recovered camera parameters of both approaches are reported in Table 1.

Figure 6 shows another example of the experiments. The camera employed is a Nikon D200 with a 20 mm Nikkor lens. A set of 30 images was acquired, including coded targets to perform an automated camera calibration. The self-calibrating bundle adjustment with and without coded targets achieved very similar results for the interior parameters (Table 2).
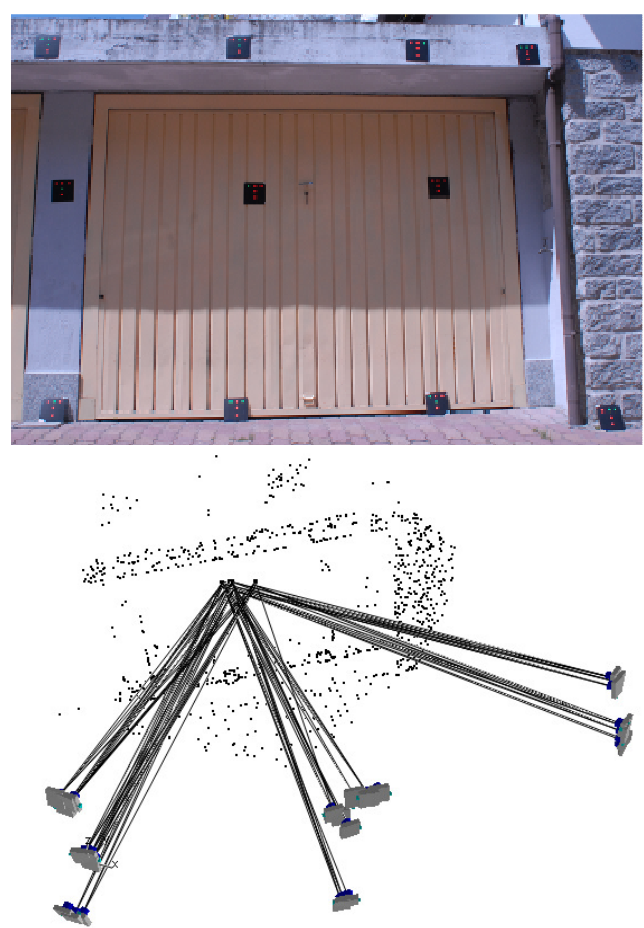

Figure 6 . The scene used for the automated camera calibration with and without coded targets (top). The camera network of the targetless solution with the recovered camera poses and sparse point cloud (bottom).

\begin{tabular}{c|cc|cc}
\cline { 2 - 5 } \multicolumn{2}{c|}{} & \multicolumn{2}{c|}{ Targets } & \multicolumn{2}{c}{ ATiPE } \\
\cline { 2 - 5 } \multicolumn{2}{c|}{ Value } & Std.dev & Value & Std.dev \\
\hline RMSE $(\mu m)$ & 1.01 & - & 3.63 & - \\
$c($ mm $)$ & 20.4367 & 0.003 & 20.4244 & 0.003 \\
$x_{0}$ & 0.0759 & 0.002 & 0.0760 & 0.002 \\
$y_{0}$ & 0.0505 & 0.003 & 0.0481 & 0.002 \\
$k_{1}$ & $2.8173 \mathrm{e}^{-4}$ & $2.605 \mathrm{e}^{-6}$ & $2.7331 \mathrm{e}^{-4}$ & $2.5102 \mathrm{e}^{-6}$ \\
$k_{2}$ & $-4.5538 \mathrm{e}^{-7}$ & $3.585 \mathrm{e}^{-8}$ & $-4.6780 \mathrm{e}^{-7}$ & $2.666 \mathrm{e}^{-8}$ \\
$k_{3}$ & $-2.7531 \mathrm{e}^{-10}$ & $1.452 \mathrm{e}^{-10}$ & $-5.8892 \mathrm{e}^{-11}$ & $9.2528 \mathrm{e}^{-11}$ \\
$p_{1}$ & $7.849 \mathrm{e}^{-6}$ & $2.113 \mathrm{e}^{-6}$ & $1.9942 \mathrm{e}^{-6}$ & $1.979 \mathrm{e}^{-6}$ \\
$p_{2}$ & $-1.6824 \mathrm{e}^{-5}$ & $2.247 \mathrm{e}^{-6}$ & $-1.4358 \mathrm{e}^{-5}$ & $1.858 \mathrm{e}^{-6}$ \\
\hline
\end{tabular}

Table 2. Results for a Nikon D200 equipped with a $20 \mathrm{~mm}$ Nikkor lens, with and without targets.

\subsection{Accuracy analysis with independent check points}

The consistence and accuracy of the first targetless calibration experiement were verified using a special testfield composed of 21 circular targets which are used as Ground Control Points (GCPs). Their 3D coordinates were measured with a theodolite Leica TS30, using three stations and a triple intersection to obtain high accuracy results. The standard deviation of the measured coordinates was $\pm 0.2 \mathrm{~mm}$ in $x$ (depth) and $\pm 0.1 \mathrm{~mm}$ in $y$ and $z$.

A photogrammetric block of 6 images was also acquired. All target centres were measured via LSM to obtain sub-pixel precisions. The photogrammetric bundle adjustment was carried out with the two sets of calibration parameters. Both photogrammetric projects were run in free-net and then transformed into the Ground Reference System (GRS) using 5 GCPs. The remaining 16 points were used as independent check points $(\mathrm{ChkP})$ to evaluate the quality of the estimation procedure. The following quantities were computed for each ChkP: 


$$
\Delta x=x_{\mathrm{GRS}}-x_{\mathrm{Photo}}, \quad \Delta y=y_{\mathrm{GRS}}-y_{\mathrm{Photo}}, \quad \Delta z=z_{\mathrm{GRS}}-z_{\mathrm{Photo}}
$$

The differences in both configurations are shown in Table 3. It can be noted that the behaviour is quite similar and the standard deviations of the differences along $x, y$ and $z$ are equivalent.

\begin{tabular}{cccc}
\cline { 2 - 4 } & \multicolumn{3}{c}{ GRS - Targets } \\
\cline { 2 - 4 } & $\Delta x$ & $\Delta y$ & $\Delta z$ \\
\hline Mean $(\mathrm{mm})$ & 0.15 & 0.59 & -0.32 \\
Std.dev $(\mathrm{mm})$ & 0.43 & 0.75 & 1.45 \\
$\operatorname{Max}(\mathrm{mm})$ & 0.83 & 1.58 & 2.22 \\
$\operatorname{Min}(\mathrm{mm})$ & -0.55 & -0.90 & -2.43 \\
\hline & \multicolumn{3}{c}{$\mathbf{G R S - A T i P E}$} \\
\cline { 2 - 4 } & $\Delta x$ & $\Delta y$ & -0.35 \\
Mean $(\mathrm{mm})$ & 0.16 & 0.61 & 1.44 \\
Std.dev $(\mathrm{mm})$ & 0.40 & 0.77 & 2.12 \\
$\operatorname{Max}(\mathrm{mm})$ & 0.81 & 1.62 & -2.42 \\
$\operatorname{Min}(\mathrm{mm})$ & -0.46 & -0.95 & \\
\hline
\end{tabular}

Table 3. Comparison between ChkP coordinates measured with a theodolite (GRS) and photogrammetric measurements with and without targets (ATiPE). The behaviour and the residuals are similar for both photogrammetric approaches.
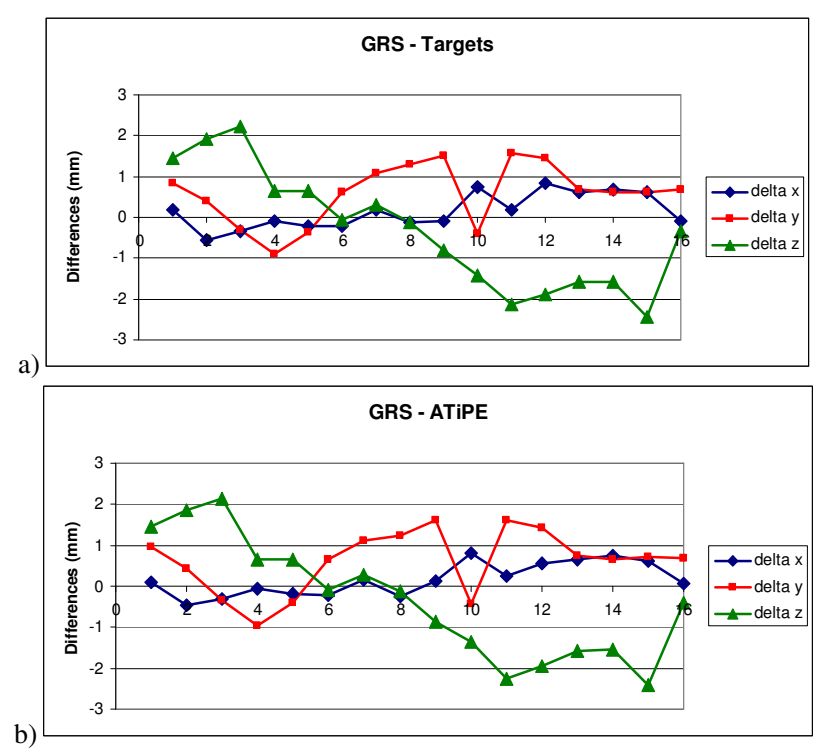

b)

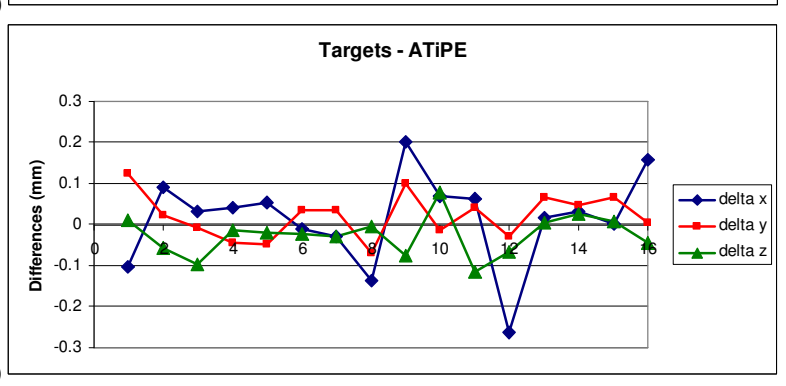

Figure 7. Graphical behaviour of the differences of the ChkP coordinates in the solution obtained using coded targets (a) and natural features extracted with ATiPE (b). The differences between the target and targetless approach are shown in (c).

It is also interesting that the differences are superior to $2 \mathrm{~mm}$ for some points. This is probably due to a residual movement of the targets during the data acquisition phase. Indeed, both reference data and images were acquired at different epochs. All targets are made of paper and probably there was a small deformation of this deformable material. This is also demonstrated by the coherence of both photogrammetric projects (Figure 7).

The coordinates of both photogrammetric projects were then compared. They confirm the consistence between these calibration datasets. A graphical visualization of the differences between the ATiPE procedure (targetless) and the estimation based on targets is shown in Figure 7.

\subsection{Analysis of covariance and correlation matrices}

The use of a free-net bundle adjustment for the estimation of the calibration parameters leads to a modification of the general form of the least squares problem. In some cases, if the network geometry is not sufficiently robust to incorporate all calibration parameters (basic interior plus the APs), the adjustment can provide highly correlated values. Therefore, a statistical evaluation of the obtained APs is always recommended (Gruen, 1981; Jacobsen, 1982; Gruen, 1985). This can be carried out by using the estimated covariance matrices and not only with the independent analysis of the standard deviations of each single unknown (Cox and Hinkley, 1976; Kendall, 1990; Sachs, 1984).

In the following, the dataset with targets is used as reference, as it can be assumed as the current state of the art for traditional photogrammetric calibrations. In particular, $\mathbf{C}_{\mathrm{T}}$ is the $8 \times 8$ covariance matrix of all calibration parameters estimated using targets. The covariance matrix with the targetless procedure is named $\mathbf{C}_{\mathrm{A}}(\mathrm{A}=\mathrm{ATiPE})$. The aim is to demonstrate that $\mathbf{C}_{\mathrm{T}}$ and $\mathbf{C}_{\mathrm{A}}$ are similar, in order to confirm the consistence of both calibrations. There exist several criteria for comparing covariance matrices, e.g. different distances $d\left(\mathbf{C}_{\mathrm{T}}, \mathbf{C}_{\mathrm{A}}\right)$ which depend on the choice of the model employed. However, it is quite complicated to understand when $d$ is small, especially if the estimated values have different measurement units. A possible solution could be to use the eigenvalues $\lambda_{i}$ of the covariance matrices, in order to obtain new diagonal matrices that can be compared (Jolliffe, 2002). According to this procedure, the directions of the principal axes of the confidence ellipsoids are given by the eigenvectors.

To check the equality of the covariance matrices, the Hotelling's test could also be used:

$$
\chi^{2}=-2 \log \frac{(\operatorname{det} \mathbf{C})^{m / 2}}{\left(\prod_{j=1}^{n} \sigma_{x j}^{2}\right)^{m / 2}}
$$

where $m$ is the number of data and $n$ the number of calibration parameters ( 8 in this case). For both matrices the ratio of the values given by Eq. 10 was estimated, obtaining a large disparity between $\mathbf{C}_{\mathrm{T}}$ and $\mathbf{C}_{\mathrm{A}}$ because of the different number of observations used during the estimation of the photogrammetric bundle solutions. To understand better the results achieved with both calibration procedures it is possible to use the correlation matrix $\mathbf{R}$. It is well-known that some calibration parameters are highly correlated, e.g. the coefficient $k_{i}$ modelling radial distortion. In addition, there is a projective coupling between $p_{1}$ and $p_{2}$ with $x_{0}$ and $y_{0}$, respectively. The experimental results provided two correlation matrices $\left(\mathbf{R}_{\mathrm{T}}, \mathbf{R}_{\mathrm{A}}\right)$ very similar, where the correlations between the listed parameter configurations are quite strong $(>0.8)$, although the targetless procedure seems slightly better (Table 4). 


\begin{tabular}{|c|c|c|c|c|c|c|c|c|}
\hline $\mathbf{R}_{\boldsymbol{T}}$ & c & $x_{p}$ & $y_{p}$ & $\mathrm{~K}_{1}$ & $\mathrm{~K}_{2}$ & $\mathrm{~K}_{3}$ & $\mathbf{P}_{1}$ & $P_{2}$ \\
\hline C & 1 & & & & & & & \\
\hline$x_{p}$ & 0.13 & 1 & & & & & & \\
\hline$y_{p}$ & -0.01 & -0.06 & 1 & & & & & \\
\hline $\mathrm{K}_{1}$ & 0.22 & 0.05 & -0.01 & 1 & & & & \\
\hline $\mathrm{K}_{2}$ & -0.27 & -0.04 & -0.05 & -0.97 & 1 & & & \\
\hline $\mathrm{K}_{3}$ & 0.24 & 0.03 & 0.07 & 0.93 & -0.99 & 1 & & \\
\hline$P_{1}$ & -0.21 & -0.91 & 0.08 & -0.11 & 0.1 & -0.09 & 1 & \\
\hline $\mathbf{P}_{2}$ & -0.01 & 0.04 & -0.93 & 0.01 & 0.03 & -0.04 & -0.06 & 1 \\
\hline $\mathbf{R}_{\mathrm{A}}$ & c & $x_{p}$ & $y_{p}$ & $\mathrm{~K}_{1}$ & $\mathrm{~K}_{2}$ & $\mathrm{~K}_{3}$ & $\mathbf{P}_{1}$ & $P_{2}$ \\
\hline c & 1 & & & & & & & \\
\hline$x_{p}$ & 0.02 & 1 & & & & & & \\
\hline$y_{p}$ & -0.04 & -0.03 & 1 & & & & & \\
\hline $\mathrm{K}_{1}$ & 0.02 & 0.02 & 0.05 & 1 & & & & \\
\hline $\mathrm{K}_{2}$ & -0.09 & -0.01 & -0.04 & -0.96 & 1 & & & \\
\hline $\mathrm{K}_{3}$ & 0.09 & 0.01 & 0.03 & 0.9 & -0.98 & 1 & & \\
\hline $\mathbf{P}_{1}$ & -0.03 & -0.86 & 0.05 & -0.02 & 0.02 & -0.02 & 1 & \\
\hline $\mathbf{P}_{2}$ & 0 & 0.03 & -0.87 & -0.09 & 0.06 & -0.05 & -0.04 & 1 \\
\hline
\end{tabular}

Table 4. The correlation matrices estimated with targets (top) and ATiPE (bottom).

For the remaining parameters, the correlations are instead correctly reduced. A consideration deserve to be mentioned: in the case of uncorrelated parameters the relationship $\operatorname{det}(\mathbf{R})=1$ must be verified. Therefore, a simple general criterion to assess the quality of these covariance matrices can be the simple comparison of the determinants:

$\operatorname{det}\left(\mathbf{R}_{\mathrm{T}}\right)=1.3 \cdot 10^{6}<\operatorname{det}\left(\mathbf{R}_{\mathrm{A}}\right)=7.1 \cdot 10^{5}$

This values are quite similar, because the matrices look quite similar. Therefore test the equality of the correlation structures, a multi-dimensional statistical analysis should be employed. Lawley's procedure (1963) requires the estimation of the following statistic:

$\chi^{2}=\left(m-1-\frac{2 n+5}{6}\right) \sum_{i=1}^{n-1} \sum_{j=i+1}^{n} r_{i j}^{2}$

where $m$ is the number of data and $n$ the number of calibration parameters (8 in this case). The application of this criterion shows that $\mathbf{C}_{\mathrm{A}}$ and $\mathbf{C}_{\mathrm{T}}$ are not equal (the ratio between the $\chi^{2}$ values is superior to 40 ), but this is mainly due to the different number of observations between the procedures (e.g. 55 3D points with the target-based method, 7,593 with ATiPE). The estimation of the ratio $\log \left(\operatorname{det}\left(\mathbf{R}\left({ }_{\mathrm{A}}\right)\right) / \log \left(\operatorname{det}\left(\mathbf{R}_{\mathrm{T}}\right)\right) \approx 32\right.$ confirms the previous results.

In summary, the statistical interpretation of the results is quite difficult because of the different numbers of input data. The comparison between different sets of calibration parameters using total station measurements is probably a better factor to check the final quality of the targetless calibration parameters.

\section{CONCLUSION}

This paper has presented a new procedure for camera calibration based on the natural texture of an object which has to be properly selected. The method can also be assumed as the initial step for a complete 3D reconstruction pipeline of some categories of objects. It is worth noting that different phases of the "reconstruction problem" can be now carried out in a fully automated way.

The proposed methodology for camera calibration is not based on targets, but it is capable of providing the unknown camera parameters values with the same theoretical accuracy of the more familiar target-based procedure. It has also been proved that the larger number of tie points extracted for computing selfcalibration gives rise to slightly smaller correlations among the parameters. But further statistical analyses should be performed. The key-point leading to a successful calibration is (i) the selection of a proper object featuring a good shape and textures and (ii) the acquisition of a set of images which results in a suitable image block geometry.

For industrial and highly-precise photogrammetric projects, the target-based camera calibration procedure will probably remain the standard solution while for many other 3D modeling applications, the presented method can be the ideal solution to speed up the entire photogrammetric pipeline, avoid targets and allow on-the-job self-calibration in a precise and reliable way.

\section{REFERENCES}

Albert, J., Maas, H.G., Schade, A. and Schwarz, W., 2002. Pilot studies on photogrammetric bridge deformation measurement. Proceedings of the 2nd IAG Symposium on Geodesy for Geotechnical and Structural Engineering: 133-140.

Amiri Parian, J., Gruen, A. and Cozzani, A., 2006. Highly accurate photogrammetric measurements of the Planck Telescope reflectors. 23rd Aerospace Testing Seminar (ATS), Manhattan Beach, California, USA, 10-12 October.

Arya, S., Mount, D.M., Netenyahu, N.S., Silverman, R. and Wu, A.Y., 1998. An optimal algorithm for approximate nearest neighbor searching fixed dimensions. Journal of the ACM, 45(6): 891-923.

Barazzetti, L. and Scaioni, M., 2009. Crack measurement: development, testing and application of an image-based algorithm. ISPRS Journal of Photogrammetry and Remote Sensing, 64: 285-296.

Barazzetti, L. and Scaioni, M., 2010. Development and implementation of image-based algorithms for measurement of deformations in material testing. Sensors: 7469-7495.

Barazzetti, L., Remondino, F. and Scaioni, M., 2010a. Extraction of accurate tie points for automated pose estimation of close-range blocks. Int. Archives of Photogrammetry, Remote Sensing and Spatial Information Sciences, Vol. 38(3A), 6 pages.

Barazzetti, L., Remondino, F. and Scaioni, M., $2010 \mathrm{~b}$. Orientation and 3D modelling from markerless terrestrial images: combining accuracy with automation. Photogrammetric Record, 25(132), pp. 356-381.

Barazzetti, L., 2011. Automatic Tie Point Extraction from Markerless Image Blocks in Close-range Photogrammetry. $\mathrm{PhD}$ Thesis, Politecnico di Milano, Milan, 155 pages.

Bay, H., Ess, A., Tuytelaars, T. and van Gool, L., 2008. Speeded-up robust features (SURF). Computer Vision and Image Understanding, 110(3): 346-359. 
Brown, D.C., 1971. Close-range camera calibration. Photogrammetric Engineering and Remote Sensing, 37(8): 855866.

Brown, D.C., 1976. The bundle adjustment - progress and prospects. Int. Arch. of Photogrammetry, Vol. 21, B3, Helsinki.

Cox, D.R. and Hinkley, D.V., 1979. Theoretical Statistics. Chapman and Hall, London. 528 pages.

Cronk, S., Fraser, C. and Hanley, H., 2006. Automated metric calibration of colour digital cameras. Photogrammetric Record, 21(116): 355-372.

D'Apuzzo, N. and Maas, H.-G., 1999. On the suitability of digital camcorders for virtual reality image data capture. In: ElHakim, S,F., Gruen, A. (Eds.), Videometrics VI, Proc. of SPIE, Vol. 3461, San Jose, USA, pp. 259-267.

Dermanis, A., 1994. The photogrammetric inner constraints. ISPRS Journal of Photogrammetry and Remote Sensing, 49(1): 25-39.

Fraser, C.S., 1992. Photogrammetric measurement to one part in a million. Photogrammetric Engineering \& Remote Sensing, 58: 305-310.

Fraser, C. S. and Shortis, M. R., 1995. Metric exploitation of still video imagery. Photogrammetric Record, 15: 107-122.

Fraser, C.S., 1996: Network design. In 'Close-range Photogrammetry and Machine Vision', Atkinson (Ed.), Whittles Publishing, UK, pp.256-282

Fraser, C.S., 1997. Digital camera self-calibration. ISPRS Journal of Photogrammetry and Remote Sensing, 52: 149-159.

Fraser, C.S. and Al-Ajlouni, S., 2006. Zoom-dependent camera calibration in close-range photogrammetry. Photogrammetric Engineering \& Remote Sensing, 72(9): 1017-1026.

Ganci, G. and Handley, H.B., 1998. Automation in videogrammetry. International Archives of Photogrammetry and Remote Sensing, 32(5): 53-58.

Granshaw, S.I., 1980. Bundle adjustment methods in engineering photogrammetry. Photogrammetric Record, 10(56): 181-207.

Gruen, A., 1981. Precision and reliability aspects in close-range photogrammetry. Photogrammetric Journal of Finland, 8(2): 117-132.

Gruen, A., 1985. Data processing methods for amateur photographs. Photogrammetric Record, 11(65): 567-579.

Gruen, A. and Beyer, H.A., 2001. System calibration through self-calibration. In 'Calibration and Orientation of Cameras in Computer Vision' Gruen and Huang (Eds.), Springer Series in Information Sciences 34, pp. 163-194.

Hartley, R.I. and Zisserman A., 2004. Multiple View Geometry in Computer Vision. Second edition. Cambridge University Press, Cambridge, 672 pages.

Gruen, A. and Huang, T., 2001. Calibration and orientation of cameras in computer vision. Springer Series in Information
Sciences 34, Springer Berlin, Heidelberg, New York, 235 pages.

Jacobsen, K., 1982. Programmgesteuerte Auswahl zusaetzlicher Parameter. Bildmessung und Luftbildwesen, 50, pp. 213-217.

Jolliffe, I. T., 2002. Principal Component Analysis, 2nd edition, Springer, 2002.

Kendall, M., 1980. Multivariate Analysis. Charles Griffin \& Company LTD, London.

Läbe, T. and Förstner, W., 2004. Geometric stability of low-cost digital consumer cameras. Int. Archives of Photogrammetry, Remote Sensing and Spatial Information Sciences, 35(5): 528535 .

Lawley, D.N., 1963. On Testing a Set of Correlation Coefficients for Equality. The Annals of Mathematical Statistics, 34(1): 149-151.

Lowe, D.G., 2004. Distinctive image features from scaleinvariant keypoints. International Journal of Computer Vision, 60(2): 91-110.

Maas, H.G. and Niederöst, M., 1997. The accuracy potential of large format still video cameras. SPIE 3174: 145-152.

Mikhail, E.M., Bethel, J. and McGlone, J.C., 2001. Introduction to Modern Photogrammetry. John Wiley \& Sons. 479 pages.

Peipe, J. and Tecklenburg, W., 2006. Photogrammetric camera calibration software - a comparison. International Archives of Photogrammetry, Remote Sensing and Spatial Information Sciences, 36(5), 4 pages

Remondino, F. and Fraser, C., 2006. Digital camera calibration methods: considerations and comparisons. International Archives of Photogrammetry, Remote Sensing and Spatial Information Sciences, 36(5): 266-272.

Sachs, L., 1984. Applied Statistics. A handbook of Techniques. Springer, New York.

Triggs, B., McLauchlan, P.F., Hartley, R. and Fitzgibbon, A., 2000. Bundle adjustment - A modern synthesis. In Vision Algorithm '99, Triggs/Zisserman/Szeliski (Eds), LNCS 1883, pp. 298-372.

Australis - www.photometrix.com.au

CLORAMA - www.4dixplorer.com

Photomodeler - www.photomodeler.com 